\title{
Association between serum ferritin level and thyroid hormones in hypothyroid pediatric patients
}

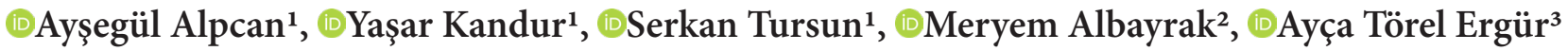 \\ ${ }^{1}$ Kırıkkale University, School of Medicine, Department of Pediatrics, Kırıkkale, Turkey \\ ${ }^{2}$ Kırıkkale University, School of Medicine, Department of Pediatric Hematology and Oncology, Kırıkkale, Turkey \\ ${ }^{3}$ Ufuk University, Faculty of Medicine, Department of Pediatric Endocrinology, Ankara, Turkey
}

Cite this article as: Alpcan A, Kandur Y, Tursun S, Albayrak M, Törel Ergür A. Association between serum ferritin level and thyroid hormones in hypothyroid pediatric patients. Anatolian Curr Med J 2021; 3(4); 300-302.

\begin{abstract}
Aim: The present study aimed to investigate the iron store status in patients with hypothyroidism due Hashimoto thyroiditis and subclinical hypothyroidism.

Material and Method: We retrospectively reviewed the medical records of patients who were followed up at our university hospital with the diagnosis of hypothyroidism due to Hashimoto thyroiditis and subclinical hypothyroidism between 2010-2021.

Results: Nineteen patients with Hashimoto thyroiditis, 17 with subclinical hypothyroidism, and 20 controls were enrolled in this study. There was no significant difference in mean age among three groups (138.3 \pm 46.8 months, $116.0 \pm 68.1$ months, $151.8 \pm 50.3$ months in groups of Hashimoto thyroiditis, subclinical hypothyroidism and controls respectively $\mathrm{p}=0.169)$. There was a significant female preponderance in HT group $(68.5 \%, \mathrm{p}=0.001)$. There was no significant difference for mean ferritin levels among groups $(\mathrm{p}=0.440)$. There was no correlation between TSH and ferritin level $(\mathrm{r}=-0.132, \mathrm{p}=0.410)$ but we found a positive correlation between ferritin and free T4 level $(\mathrm{r}=0.340, \mathrm{p}=0.030)$.

Conclusion: We found a positive relationship between ferritin and thyroxine hormone that made us think that iron supplementation may be effective in the treatment of hypothyroidism. But this hypothesis warrants further prospective and experimental studies.

Keywords: Children, ferritin, hypothyroidism
\end{abstract}

\section{INTRODUCTION}

Iron deficiency may impair thyroid functions. Previous studies have suggested that iron deficiency may impair thyroid hormone metabolism. These studies showed that in iron-deficient-anemic subjects, serum T3 and T4 levels were significantly decreased $(1,2)$. Although some studies showed a correlation between ferritin level and hypothyroidism, $(3,4)$ yet there is no agreement on this subject and the study groups were not composed of patients with thyroid disorders. The present study aimed to investigate the iron store status in patients with hypothyroidism, Hashimoto thyroiditis (HT) and subclinical hypothyroidism (SH).

\section{MATERIAL AND METHOD}

We retrospectively reviewed the medical records of patients who were followed up at our university hospital with the diagnosis of hypothyroidism, $\mathrm{HT}$ and $\mathrm{SH}$ between 2010-2021. The control group was selected from healthy children aged 1 month to 17 years who were examined at the healthy child division and whose serum ferritin levels and thyroid function tests were measured. The demographic characteristics, medical history, and laboratory findings of the patients were collected from the patients' medical records. Patients with hyperthyrodism, and congenital hypothyroidism were excluded. Patients with ultrasonographic findings and the presence of antibodies against thyroid peroxidase (TPO antibodies) confirmed the diagnosis of $\mathrm{HT}$ (5). $\mathrm{SH}$ is defined as isolated hyperthyrotropinemia with high serum TSH levels, and normal free T4 concentrations (6). The ethics committee approval of the study was obtained from the Kurıkkale University Non-interventional Researchs Ethics Committee (Date: 30.06.2021, Decision No: 2021.05.01). All procedures were carried out in accordance with the ethical rules and the principles of the Declaration of Helsinki. 


\section{Statistical Analysis}

All statistical analyses were performed using SPSS (Statistical Package for the Social Sciences) version 20.0 software (SPSS Inc.,USA). We presented categorical variables in the form of frequency and percentage and continuous variables in the form of mean and standard deviation (SD). The continuous variables were compared with One Way ANOVA variance analysis test among three groups. Binary comparisons were done with post hoc Tukey and Duncan tests. Chi-square test and Fisher's exact test were used to compare categorical variables among three groups where applicable. The possible correlation between thyroid hormones and ferritin level was analysed with Spearman's rank correlation test. A two-sided $p$ value of less than 0.05 was considered statistically significant.

\section{RESULTS}

Nineteen patients with HT, 17 with SH, and 20 controls were enrolled in this study. The demographic chracteristics and laboratory parameters of all study population were presented on Table. There was no significant difference in mean age among three groups (138.3 \pm 46.8 months, $116.0 \pm 68.1$ months, $151.8 \pm 50.3$ months in groups of HT, SH and controls respectively $\mathrm{p}=0.169)$. There was a significant female preponderance in HT group (68.5\%, p=0.001). The TSH levels in HT and $\mathrm{SH}$ were significantly greater and the free $\mathrm{T} 4$ levels were lower than the control group as expected (Table). There was no significant difference for mean ferritin levels among groups $(\mathrm{p}=0.440)$. Nor hemoglobin neither mean corpuscular volume levels showed significant difference among three groups. Also there was no significant difference among groups for parameters of mean vitamin B12 $(p=0.555)$ and folic acid levels $(p=0.879)$.

There was no correlation between TSH and ferritin level $(\mathrm{r}=-0.132, \mathrm{p}=0.410)$ but we found a positive correlation between ferritin and free T4 level $(r=0.340, p=0.030)$. Also there was a positive correlation between hemoglobin and ferritin level as expected $(\mathrm{r}=0.538, \mathrm{p}=<0.001)$.

\section{DISCUSSION}

In our study, we observed female preponderance in HT patients compared to $\mathrm{SH}$ and controls and we observed a positive correlation between ferritin and free T4 level. The iron load can be evaluated with plasma ferritin level that is a simple, accessible and inexpensive method (7). Although Shamshirsaz et al. (8) found no correlation between ferritin level and hypothyroidism, Chirico et al. (9) found such a correlation. But, ferritin level did not show a significant difference between controls and patients with thyroid disorder in Chirico's study similar to our results.

Sakata et al. (10) found that ferritin levels were higher in patients with $\mathrm{SH}$ than in controls. But in our study, the ferritin level was slightly higher in the SH group but it was insignificant. In our study population we found lower levels of ferritin in the HT group in comparison to controls but it was insignificant. We thought that this result may arise from the high percentage of female patients in this group whose ferritin levels were under the effect of menorrhagia.

Hess et al. (11) have shown that thyroid peroxidase activity is significantly decreased in iron deficiency anemia. On the contrary Tienboon et al. (12) found statistical difference in thyroid hormones of the irondeficient anemic children. And they found a positive and significant correlation between the serum ferritin level and the free T4 levels similar to our results. This finding may be the result of deficiency in iron-dependent enzymes such as thyroid peroxidase that have had influence on thyroid metabolism.

We observed that the free T4 level was lower in HT group than in the control group but it was an insignificant difference at thyroid-stimulating hormone level. Moreover Takamatsu et al. (13) showed that serum ferritin levels increased in all hypothyroid patients with HT when euthyroidism was achieved with L-T4 therapy. Nevertheless we thought that ferritin level had an effect on free T4 level.

\begin{tabular}{|c|c|c|c|c|}
\hline Parameters & Hashimoto thyroiditis $(\mathrm{N}=19)$ & Subclinical hypothyroidsm $(\mathrm{N}=17)$ & Control $(\mathrm{N}=20)$ & P value \\
\hline Age (month) & $138.3 \pm 46.8$ & $116.0 \pm 68.1$ & $151.8 \pm 50.3$ & 0.169 \\
\hline Female gender $\mathrm{n}(\%)$ & $15(68.5)$ & $7(42)$ & $10(50)$ & 0.001 \\
\hline TSH (uIU / mL) & $17.2 \pm 6.8$ & $7.3 \pm 3.1$ & $2.7 \pm 0.9$ & 0.043 \\
\hline Free T4 (ng/dL) & $1.06 \pm 0.24$ & $1.57 \pm 0.71$ & $1.31 \pm 0.17$ & 0.003 \\
\hline Ferritin $(\mathrm{ng} / \mathrm{mL})$ & $30.8 \pm 22.1$ & $44.0 \pm 29.1$ & $42.2 \pm 11.7$ & 0.440 \\
\hline $\mathrm{Hg}(\mathrm{g} / \mathrm{dL})$ & $13.2 \pm 1.6$ & $13.4 \pm 1.7$ & $14.0 \pm 1.7$ & 0.332 \\
\hline MCV (fL) & $78.2 \pm 7.7$ & $79.4 \pm 4.6$ & $83.1 \pm 7.0$ & 0.08 \\
\hline Vitamin B12 (pg/mL) & 567 & $325 \pm 165$ & $373 \pm 139$ & 0.555 \\
\hline Folic acid (ng/mL) & $8.4 \pm 4.7$ & $9.2 \pm 4.1$ & $9.1 \pm 2.7$ & 0.879 \\
\hline
\end{tabular}

The values are presented as mean \pm SD and $n(\%)$. Hg: Hemoglobin, TSH:Thyroid stimulating hormone, T4: levothyroxine, MCV: mean corpuscular volume. 
We found no significant difference between ST and HT groups for mean ferritin level. Ferritin is also an acutephase reactant that is elevated in many conditions sush as infection (viral and bacterial), autoimmunity, stem cell transplant, malignancy and renal disease (14). However in some auto-immune inflammatuary diseases such as the rheumatoid arthritis (RA), serum ferritin may not be increased (15). We suggested that like RA, HT is a disease where ferritin increment is not observed as an acute phase reactant reaction. There may be different pathogenic pathways to explain this condition in HT.

The insignificant difference of vitamin $\mathrm{B} 12$ and folate levels among our groups may also showed us that thyroid hormones had no effect on gastrointestinal absorption. Studies of intestinal absorptive functions in 11 hyperthyroid and 14 hypothyroid patients showed that steatorrhoea was common in hyperthyroid patients and rare in hypothyroid patients (16). Since our study group composed of hypothyroid patients we found no difference for vitamin B12 and folate levels as expected.

The present study has the following limitations. Firstly, because of its retrospective nature and reliance on medical records, some of the investigated data were not available for all patients such serum iron levels. Secondly the small sample study population limits the power of study. And our single-center study design does not project the whole population. So that our findings should be confirmed by further multicenter prospective studies with a larger sample size.

\section{CONCLUSION}

We found a positive relationship between ferritin and thyroxine hormone that made us think that iron supplementation may be effective in the treatment of hypothyroidism. The underlying mechanisms of ferritin role in the modulation of thyroid functions and its specific feature except acute phase reactant for HT, warrant further prospective and experimental studies.

\section{ETHICAL DECLARATIONS}

Ethics Committee Approval: The ethics committee approval of the study was obtained from the Kirıkkale University Non-interventional Researchs Ethics Committee (Date: 30.6.2021, Decision No: 2021.05.01).

Informed Consent: Because the study was designed retrospectively, no written informed consent form was obtained from patients.

Referee Evaluation Process: Externally peer-reviewed.

Conflict of Interest Statement: The authors have no conflicts of interest to declare.

Financial Disclosure: The authors declared that this study has received no financial support.
Author Contributions: All of the authors declare that they have all participated in the design, execution, and analysis of the paper, and that they have approved the final version.

\section{REFERENCES}

1. Martinez-Torres C, Cubeddu L, Dillman E, et al. Effect of exposure to low temperature on normal and iron-deficient subjects. Am J Physiol 1984; 246: R380-3.

2. Beard JL, Borel MJ, Deer J. Impaired thermoregulation and thyroid function in iron deficiency anemia. Am J Clin Nutr 1990; 52: 813-9.

3. Eshragi P, Tamaddoni A, Zarifi $\mathrm{K}$, et al. Thyroid function in major thalassemia patients: Is it related to height and chelation therapy? Caspian J Intern Med 2011; 2: 189.

4. İpek İÖ, Kaçmaz E, Bozaykut A, Sezer RG, Seren L, Paketçi C. The effect of iron deficiency anemia on plasma thyroid hormone levels in childhood. Turk Arch Pediatr. 2011;46:122-5.

5. Caturegli P, De Remigis A, Rose NR. Hashimoto thyroiditis: clinical and diagnostic criteria. Autoimmun Rev. 2014;13(45):391-7

6. Gallizzi R, Crisafulli C, Aversa T, et al. Subclinical hypothyroidism in children: is it always subclinical? Ital J Pediatr 2018; 44: 25.

7. Tiwari R, Saharia GK, Mangaraj M. Evaluation of serum ferritin and anti-thyroid peroxidase antibody status in newly diagnosed subclinical cases of hypothyroidism. Endocr Metab Immune Disord Drug Targets 2020.

8. Shamshirsaz AA, Bekheirnia MR, Kamgar M, et al. Metabolic and endocrinologic complications in beta-thalassemia major: a multicenter study in Tehran. BMC Endocr Disord 2003; 3: 4.

9. Chirico V, Antonio L, Vincenzo S, et al. Thyroid dysfunction in thalassaemic patients: ferritin as a prognostic marker and combined iron chelators as an ideal therapy. Eur J Endocrinol 2013; 169: 785-93.

10. Sakata S,Nagai K, Maekawa $H$, et al. Serum ferritin concentration in subacute thyroiditis. Metabolism 1991; 40: 683-8.

11. Hess SY, Zimmermann MB, Arnold M, Langhans W, Hurrell RF. Iron deficiency anemia reduces thyroid peroxidase activity in rats. J Nutr 2002; 132: 1951-5

12. Tienboon P, Unachak K. Iron deficiency anaemia and thyroid function. Asia Pac J Clin Nutr 2003; 12: 198-202.

13. Takamatsu J, Majima M, Miki K, Kuma K, Mozai T. Serum ferritin as a marker of thyroid hormone action on peripheral tissues. J Clin Endocrinol Metab. 1985; 614: 672-6.

14. Knovich MA, Storey JA, Coffman LG, Torti SV, Torti FM. Ferritin for the clinician. Blood Rev. 2009; 23: 95-104.

15. Kumon Y, Suehiro T, Nishiya K, Hashimoto K, Nakatani K, Sipe JD. Ferritin correlates with C-reactive protein and acute phase serum amyloid A in synovial fluid, but not in serum. Amyloid 1999; 6: 130-5.

16. Misra GC, Bose SL, Samal AK. Malabsorption in thyroid dysfunctions. J Indian Med Assoc 1991; 89: 195-7. 